\title{
Diagnostic accuracy of a duplex real-time reverse transcription quantitative PCR assay for detection of African horse sickness virus
}

\author{
Alan J. Guthrie a,*, N. James MacLachlan ${ }^{\mathrm{b}, \mathrm{c}}$, Christopher Joone ${ }^{\mathrm{a}}$, Carina W. Lourens ${ }^{\mathrm{a}}$, Camilla T. Weyer ${ }^{\mathrm{a}}$, \\ Melvyn Quan ${ }^{c}$, Mpho S. Monyai ${ }^{a}$, Ian A. Gardner ${ }^{\mathrm{d}}$
}

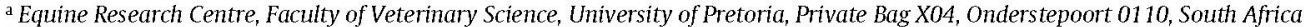

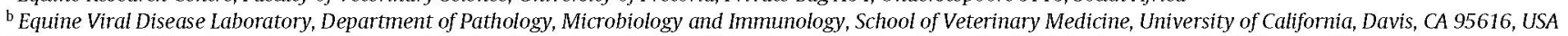

${ }^{c}$ Department of Veterinary Tropical Diseases, Faculty of Veterinary Science, University of Pretoria, Private Bag X04, Onderstepoort 0110, South Africa

$\mathrm{d}$ Department of Health Management, Atlantic Veterinary College, Charlottetown, Prince Edward Island C1A 4P3, Canada

Keywords:

Diagnostic sensitivity

Diagnostic specificity

STARD

RT-qPCR

African horse sickness virus

\section{A B S T R A C T}

Blood samples collected from 503 suspect cases of African horse sickness (AHS) and another 503 from uninfected, unvaccinated South African horses, as well as 98 samples from horses from an AHS free country, were tested with an AHS virus (AHSV) specific duplex real-time reverse transcription quantitative PCR (RT-qPCR) assay and virus isolation (VI). The diagnostic sensitivity and specificity of this AHSV RTqPCR assay and VI were estimated using a 2-test 2-population Bayesian latent class model which made no assumptions about the true infection status of the tested animals and allowed for the possibility of conditional dependence (correlation) in test results. Median diagnostic sensitivity and specificity of the AHSV RT-qPCR were $97.8 \%$ and $99.9 \%$, respectively. Median diagnostic specificity of virus isolation was $>99 \%$ whereas the estimated diagnostic sensitivity was $44.2 \%$. The AHSV RT-qPCR assay provides for rapid, high-throughput analysis of samples, and is both analytically and diagnostically sensitive and specific. This assay is potentially highly useful for demonstrating freedom or infection of horses with AHSV, thus it is appropriate that its reproducibility be evaluated in other laboratories as a global standard for detection of AHSV.

\section{Introduction}

African horse sickness virus (AHSV), a member of the genus Orbivirus, family Reoviridae, is the aetiological agent of African horse sickness (AHS), a non-contagious, insect-transmitted disease of equids. AHS is endemic throughout much of sub-Saharan Africa, but substantial incursions have occurred previously into North Africa, the Iberian Peninsula, the Middle East and the Indian subcontinent (Guthrie and Quan, 2009; MacLachlan and Guthrie, 2010). Nine serotypes of AHSV have been described (Howell, 1962; McIntosh, 1958). The genome consists of 10 double stranded RNA segments, encoding 7 structural (VP1-VP7) and 4 non-structural (NS1, NS2, NS3, NS3A) proteins (Grubman and Lewis, 1992; Roy et al., 1994). Genome segment 7 (S7) encodes the inner capsid protein VP7, which is highly conserved among the 9 AHSV serotypes (Quan et al., 2010) and is the basis for several antigen (Hamblin et al., 1991; Laviada et al., 1992), antibody (Hamblin et al., 1990; Kweon et al., 2003; Maree and Paweska, 2005; Wade-Evans et al., 1993), and nucleic-acid (Agüero et al., 2008; Fernández-Pinero et al., 2008;

* Corresponding author. Tel.: +27 125298068 ; fax: +27 125298301 .

E-mail addresses: alan.guthrie@up.ac.za, alang@icon.co.za (A.J. Guthrie).
Laviada et al., 1997; Quan et al., 2010; Zientara et al., 1993, 1995) based diagnostic assays. AHS is a World Organisation for Animal Health (OIE)-listed disease because of its associated high mortality and potential for rapid international spread (Anon, 2012c). An outbreak of AHS in a naïve horse population can be devastating, with a cumulative mortality rate of up to $95 \%$ (Coetzer and Guthrie, 2004).

At least 3 reverse transcriptase quantitative polymerase chain reaction (RT-qPCR) assays targeting the $\mathrm{S7}$ gene (Agüero et al., 2008; Fernández-Pinero et al., 2008; Quan et al., 2010) and 2 other assays targeting the S8 gene (encoding NS2) (Monaco et al., 2011; Quan et al., 2010) of AHSV have been described and characterised recently. These studies provide data on the linearity, efficiency, limit of detection (analytical sensitivity) and analytical specificity of each assay. However, the performance of each of these assays has been evaluated only using cell-culture derived reference or putative prototype strains of AHSV, and only a limited number of tissue or blood samples from natural or experimental cases of AHS. To date, the sensitivity and specificity of these assays have not been investigated on field diagnostic samples and therefore none of the described assays have been included in the OIE Terrestrial Manual (Anon, 2012b) as prescribed tests for international trade purposes.

The aim of this study was to estimate the diagnostic accuracy of an optimised and well-characterised RT-qPCR assay for detection 
of AHSV. It is proposed that this assay be used internationally for the following purposes: (1) to demonstrate freedom from AHSV infection in a defined population of horses; (2) for certification of freedom from infection or presence of AHSV in individual horses for trade/movement purposes; and (3) as confirmation of diagnosis of suspect or clinical AHS cases using whole blood samples collected from horses, following the guidelines for reporting studies of diagnostic accuracy (Standards for Reporting of Diagnostic Accuracy, STARD) (Bossuyt et al., 2003; Gardner et al., 2011). The diagnostic sensitivity and specificity of this AHSV RT-qPCR assay and that of conventional virus isolation were estimated using a 2-test 2-population Bayesian latent class model, which made no assumptions about the true infection status of the tested animals.

\section{Materials and methods}

\subsection{AHSV RT-qPCR group specific assay and virus isolation}

A previously published group-specific RT-qPCR assay developed and optimised in our laboratory using VP7-specific primers and probe and field and laboratory strains of the 9 serotypes of AHSV (Quan et al., 2010) was adapted for use in this study. In addition, proprietary Xeno4 primers and probe to target XenoRNA were included as a synthetic external control (EC) for all samples (Fang et al., 2007). The concentrations of primers were limited; for the AHSV assay the forward (AGA GCT CTT GTG CTA GCA GCC T) and reverse (GAA CCG ACG CGA CAC TAA TGA) primer concentrations were $200 \mathrm{nM}$ and the probe (FAM-TGC ACG GTC ACC GCT-MGB) concentration was $120 \mathrm{nM}$, whilst for the Xeno4 (EC) assay the concentration of both primers was $250 \mathrm{nM}$ and the probe (labelled with VIC) concentration was $200 \mathrm{nM}$. The primer-probe mixture was supplied by the manufacturer (Applied Biosystems part number $4445067)$ at a $25 \times$ concentration.

All extractions were performed on a Kingfisher 96 magnetic particle processor (Thermo Fisher Scientific Inc.) using the MagMAX ${ }^{\mathrm{TM}}$ pathogen RNA/DNA kit (Applied Biosystems part number 4462359) according to the manufacturer's recommendations with slight modifications. Briefly, $100 \mu \mathrm{l}$ of blood were added to $20 \mu \mathrm{l}$ of magnetic bead mix in a 96-well (deep well) plate and then vortexed at $1000 \mathrm{rpm}$ for $60 \mathrm{~s}$. A volume of $400 \mu \mathrm{l}$ of lysis buffer (which included 20,000 copies of XenoRNA which is supplied as part of the VetMax ${ }^{\mathrm{TM}}$-Plus one-step RT-PCR Kit (Applied Biosystems part number 4415328) was then added to each well and the plate was again vortexed. The plate was then transferred to the magnetic particle processor and processed using a modification of the 4462359_DW_HV script in which the final 3 min step of the elution of nucleic acid into $90 \mu \mathrm{l}$ of elution buffer was performed at $95^{\circ} \mathrm{C}$ in order to denature dsRNA in the eluate. The plate was immediately sealed using foil and frozen at $-20^{\circ} \mathrm{C}$ for $5 \mathrm{~min}$. Forty-four samples were processed in duplicate on each plate. Blood from an AHSV uninfected, unvaccinated horse was placed in 5 randomly placed wells on each plate to serve as negative controls.

RT-qPCR was performed by adding $20 \mu$ l of VetMax ${ }^{\mathrm{TM}}$-Plus onestep RT-PCR mastermix, including primers and probes, to each well on a 96-well PCR plate. Five microliters of the denatured eluate was transferred from the elution plate to the PCR plate. An AHSVnegative template and low- and high-positive AHSV controls were then added to wells F12, G12 and H12, respectively, of each plate. The plate was then sealed with a transparent plate sealer and the RT-qPCR was performed following the manufacturer's recommendations on a StepOnePlus ${ }^{\mathrm{TM}}$ Real-Time PCR System (Applied Biosystems). Samples were classified as positive if the normalised fluorescence for the AHSV assay exceeded a 0.1 threshold within 40 PCR cycles in both replicates of each sample. Samples were classified as negative if the normalised fluorescence for the Xeno4 assay exceeded a 0.1 threshold within 33 PCR cycles and if the normalised fluorescence for the AHSV assay did not exceed a 0.1 threshold within 40 PCR cycles in both replicates of each sample. Samples in which there was discordance in interpretation between the replicates were retested. The quantification cycle (Cq) was defined as the cycle number during which the fluorescence threshold was reached. Virus isolation (VI), which is considered the reference method for confirmation of infection of horses with AHSV, was performed on BHK21 cells as recommended in the OIE Terrestrial Manual (Anon, 2012b) and described previously (Quan et al., 2008, 2010).

\subsection{Determination of assay linear range, efficiency, limit of detection, intra- and inter-run variability and cross-contamination}

The linear range of the entire assay (i.e. nucleic acid extraction and AHSV RT-qPCR) was determined using undiluted horse blood obtained from an experimental case of AHS and a series (7) of 10-fold (1:10; v:v) dilutions using blood obtained from the same horse prior to infection. Each dilution was tested 5 times in a single run. PCR efficiencies were determined by the formula: PCR efficiency $(\%)=100 \times\left(10^{1 / \text { slope }}-1\right)$. Furthermore, a twofold dilution series consisting of 15 separate dilutions was made using the $10^{-3}$ dilution of AHSV-positive blood prepared above and which covered the non-linear range was extracted 5 times in each of 5 independent runs and tested by AHSV RT-qPCR analysis. The results of these analyses were used to calculate the limit of detection (i.e. input concentration giving a positive RT-qPCR result in $95 \%$ of the repeats (Burns and Valdivia, 2008)). The inter-run, intra-run and total standard deviations (SD) were calculated by the formulas: inter-run SD, standard deviation of the means of all runs; intra-run SD, mean of the standard deviations of all runs; total SD, standard deviation of all replicates. The total coefficient of variation (CV) was calculated by the formula: total $\mathrm{CV}=$ total $\mathrm{SD} /($ mean $\mathrm{Cq}-\mathrm{value}$ of all replicates).

To confirm the absence of cross-contamination in the assay, 24 AHSV positive and 72 negative horse blood samples were arranged in a chequerboard pattern (Fang et al., 2007). The samples were extracted automatically and evaluated for cross-contamination by AHSV RT-qPCR analysis on two different days.

\subsection{Study design}

A total of 503 equine blood samples collected from AHS suspect cases submitted to our laboratory between 1 January 2011 and 31 May 2012 were used to validate the assay. Only blood samples from pyrexic horses with one or more signs typical of AHS (Guthrie and Quan, 2009) were included. On receipt in the laboratory, two $1 \mathrm{ml}$ aliquots of the original sample were transferred to cryotubes and stored at $-80^{\circ} \mathrm{C}$ prior to AHSV RT-qPCR during May-July, 2012. Virus isolation was performed by the same individual (CWL) on the remainder of the original sample as soon after receipt as possible. Individuals performing and interpreting the virus isolation and RTqPCR assays were blinded as to the origin of the samples and the results of the other test. The outcomes of the independent tests were entered into a database and decoded by a third person who was not responsible for performing either test.

Blood samples were also collected from two separate healthy populations of horses that were not vaccinated against AHS and were highly unlikely to have been exposed to natural infection with AHSV. Whole blood samples were collected in EDTA in January 2012 from 1707 Thoroughbred foals born and resident since birth in the AHS-controlled area in the Western Cape Province of South Africa. These foals were all born between July 2011 and December 2011 and had not been vaccinated against AHS prior to sample 
Table 1

Inter- and intra-run variation for 15 twofold dilutions of AHSV-infected horse blood.

\begin{tabular}{|c|c|c|c|c|c|}
\hline \multirow{2}{*}{$\begin{array}{l}\text { Samples } \\
\log _{10} \text { dilution }\end{array}$} & \multicolumn{5}{|c|}{ Results (Cq) } \\
\hline & Mean & Inter-run SD & Intra-run SD & Total SD & Total CV \% \\
\hline-3.30 & 28.97 & 0.88 & 0.37 & 0.93 & 3.2 \\
\hline-3.60 & 29.59 & 0.39 & 0.16 & 0.39 & 1.3 \\
\hline-3.90 & 30.36 & 0.19 & 0.16 & 0.24 & 0.8 \\
\hline-4.20 & 31.15 & 0.10 & 0.11 & 0.14 & 0.5 \\
\hline-4.51 & 31.99 & 0.20 & 0.22 & 0.28 & 0.9 \\
\hline-4.81 & 32.85 & 0.10 & 0.30 & 0.29 & 0.9 \\
\hline-5.11 & 33.68 & 0.20 & 0.33 & 0.37 & 1.1 \\
\hline-5.41 & 34.70 & 0.34 & 0.78 & 0.79 & 2.3 \\
\hline-5.71 & 36.07 & 0.66 & 1.66 & 1.77 & 4.9 \\
\hline-6.01 & 37.49 & 1.11 & 1.43 & 1.70 & 4.5 \\
\hline-6.31 & 39.02 & 0.69 & 1.04 & 1.19 & 3.1 \\
\hline-6.61 & 39.46 & 0.46 & 0.91 & 1.13 & 2.9 \\
\hline-6.91 & 39.15 & 0.80 & 1.10 & 1.45 & 3.7 \\
\hline-7.21 & 39.63 & 0.39 & 0.56 & 0.77 & 1.9 \\
\hline-7.52 & 40.00 & 0.00 & 0.00 & 0.00 & 0.0 \\
\hline
\end{tabular}

The line between the -5.41 and $-5.71 \log _{10}$ dilution represents the limit of detection.

collection. Of these foals, 445 were resident within the AHS Surveillance Zone of South Africa and the remaining 1262 were resident within the AHS Protection Zone of South Africa. All 445 samples from the horses within the AHS Surveillance Zone were tested whilst a total of 58 samples were randomly selected from foals resident within the AHS Protection Zone. An on-going surveillance programme for AHS is in place in the AHS-controlled area of South Africa and there was no evidence of AHSV circulation in the area for at least 60 days prior to, or since the birth of any of the foals. The second population included 98 whole blood samples collected in EDTA from horses that were randomly selected from samples provided by the Central Veterinary Research Laboratory (CVRL), Dubai, United Arab Emirates. The horses were of various ages, breeds and sexes and had all been resident in Dubai, United Arab Emirates for at least 60 days prior to collection. These two groups of samples were processed as described above and were designated as the South African and CVRL healthy horse populations, respectively. Reporting of study findings was done in accordance with STARD guidelines (Gardner et al., 2011) (see Supplemental data S1).

Supplementary data associated with this article can be found, in the online version, at http://dx.doi.org/10.1016/j.jviromet. 2012.12.014.

\subsection{Statistical analyses}

The sensitivity and specificity of the AHSV RT-qPCR and virus isolation for detection of AHSV nucleic acid and AHSV, respectively, in whole blood samples were estimated using a 2-test 2-population Bayesian latent class model that allowed for conditional dependence (correlation) among test results, as previously described (Branscum et al., 2005; Georgiadis et al., 2003). Latent class methods are accepted as a valid approach by the OIE for estimation of sensitivity and specificity (Anon, 2012a). A Bayesian model does not depend on a reference test (sometimes termed a "gold standard"), although prior knowledge about the test accuracy can be readily incorporated into the analysis through the use of informative priors. Because the 2-test 2-population dependence model requires informative prior information on 2 parameters to ensure identifiability, we used a highly informative beta $(9999,1)$ prior for the specificity of VI. This prior allowed for approximately 1 false-positive VI result (possible cross-contamination) in 10,000 non-infected samples. The second informative prior (beta $(1,100)$ ) was for AHSV infection prevalence in the assumed AHSV-free population. This constrained prevalence to be low, if AHSV was present but not detected. Non-informative (beta $(1,1)$ priors, which allowed all values between 0 and 1 to have equal probability, were used for all other parameters. A sensitivity analysis was done using a simpler conditional independence model in which beta $(1,1)$ priors were used for all parameters. Models were run in WinBUGS 1.4.3 (Lunn et al., 2000) (available at http://www.mrc-bsu.cam.ac.uk/bugs/winbugs/contents.shtml) with the first 5000 iterations discarded and the next 50,000 iterations used for posterior inferences (medians and 95\% probability intervals for sensitivity, specificity and prevalence). Model convergence was assessed by visual inspection of trace plots of iterated values and running multiple chains from dispersed initial values.

\section{Results}

\subsection{Assay linear range, efficiency, limit of detection and intra- and inter-run variability}

Linear regression analysis indicated that the AHSV RT-qPCR assay had a linear range extending from the $10^{-6}$ dilution to the $10^{0}$ dilution of the positive control sample with an $R^{2}>0.99$. Based on the slope, the efficiency of the assay was calculated to be $102 \%$ (Fig. 1). The limit of detection (i.e. input concentration giving a

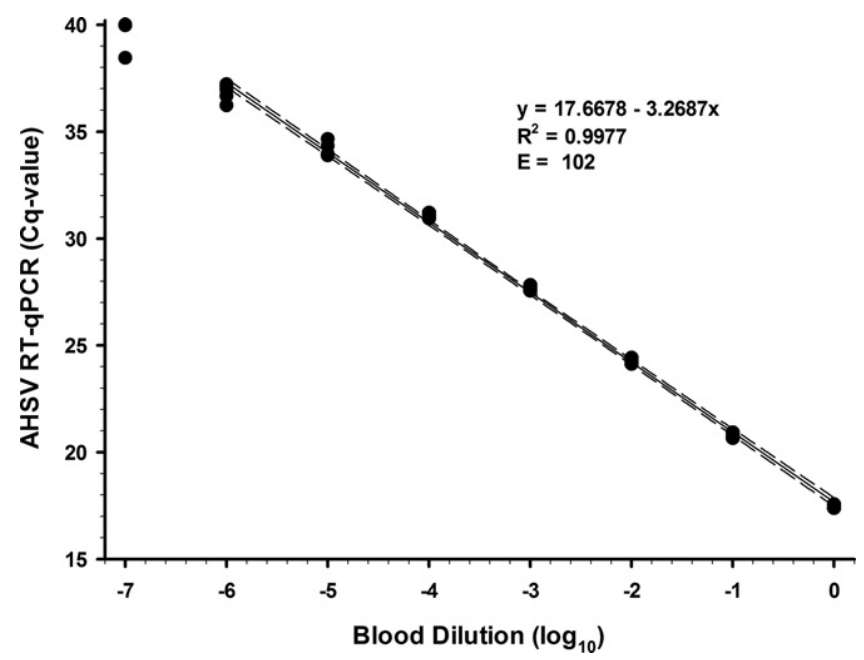

Fig. 1. Scatter plot with the regression line (solid line) and confidence interval (dashed line) of a 10-fold (v:v) dilution series of an AHSV-positive horse blood with a viral load covering the linear range. Each dilution was tested 5 times in a single run. 


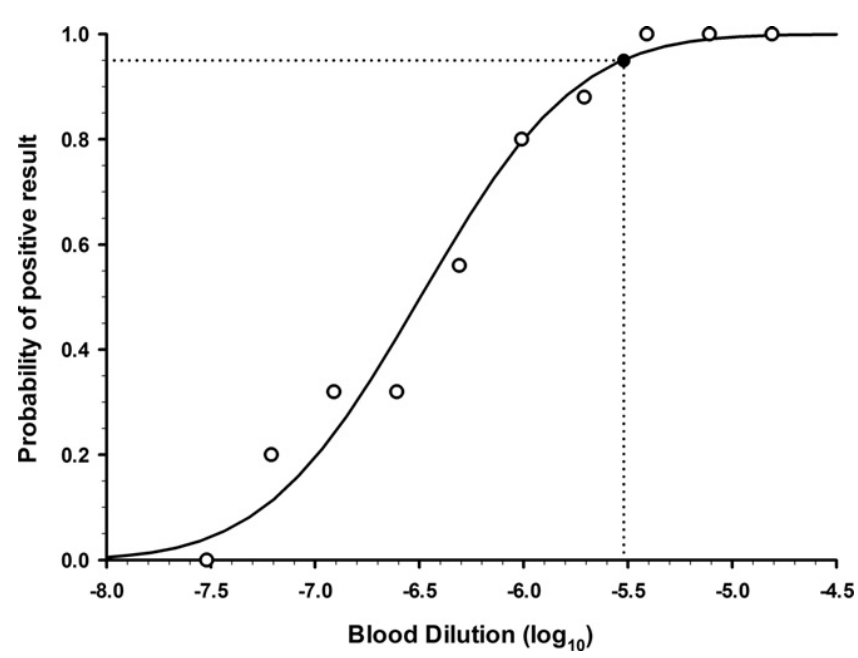

Fig. 2. Probit analysis of a twofold dilution series of an AHSV-positive horse blood with a viral load covering the non-linear range. The extraction was repeated 25 times. The $95 \%$ limit of detection is indicated with dashed lines.

positive result in $95 \%$ of the replicates) was determined by probit analysis to be at a dilution of $3.02 \times 10^{-6}$ (Fig. 2), which corresponded to a $\mathrm{Cq}$ of 35.71 . The mean results and the variations for each dilution are summarised in Table 1. Both intra- and inter-run standard deviations (SD) were low with maxima at $1.11 \mathrm{Cq}$ and $1.66 \mathrm{Cq}$, respectively. The coefficient of variation (CV) ranged from $0 \%$ to $4.9 \%$, indicating low variation between different repetitions and different runs. The CV increased towards and beyond the limit of detection. The $\mathrm{CV}$ reached a minimum at the final dilution as all results were negative. The mean Cq for the XenoRNA EC assay was $29.28 \pm 0.57$ over the 25 separate runs of the 15 dilutions of positive blood. The positive samples on the chequerboard plate gave mean Cq values of $26.75 \pm 0.18$. The XenoRNA EC assay gave a mean Cq of $29.58 \pm 0.43$. No viral RNA was detected in the negative samples in each of the two runs, and no cross-contamination was detected.

\subsection{Diagnostic sensitivity and specificity}

The results of the AHSV RT-qPCRs and VI for the AHS-suspect (clinically suspicious) horses and the healthy horse populations are summarised in Tables 2 and 3, respectively. The 156 samples that tested positive on AHSV RT-qPCR and VI included all 9 serotypes of AHSV with the number of individual isolates of each serotype ranging from 4 for serotype 9 to 58 for serotype 8 .
Table 2

Data table for AHS-suspect horses included in the analysis of sensitivity and specificity of the AHSV RT-qPCR (PCR) and virus isolation (VI) assays for detection of AHSV in blood samples.

\begin{tabular}{lrcc}
\hline Test results & VI+ & VI- & Total \\
\hline PCR+ & 156 & 184 & 340 \\
PCR - & 0 & 163 & 163 \\
Total & 156 & 347 & 503 \\
\hline
\end{tabular}

Table 3

Data table for AHSV-uninfected horses included in the analysis of sensitivity and specificity of the AHSV RT-qPCR (PCR) and virus isolation (VI) assays for detection of AHSV in blood samples.

\begin{tabular}{|c|c|c|c|}
\hline Test results & $\mathrm{VI}+$ & VI- & Total \\
\hline PCR+ & 0 & 0 & 0 \\
\hline PCR- & 0 & $503(98)$ & $503(98)$ \\
\hline Total & 0 & $503(98)$ & 503 (98) \\
\hline
\end{tabular}

Numbers in parentheses represent samples received from CVRL, Dubai, UAE.

Estimates of the sensitivity and specificity of the AHSV RT-qPCR and VI are summarised in Table 4 . The median specificity of both assays was $>99.3 \%$. The median sensitivity of the AHSV RT-qPCR in the dependence model was $>97.8 \%$ whereas the median sensitivity of VI was approximately $44.2 \%$. When the data were reanalysed using the conditional independence model and non-informative priors, specificity estimates only changed minimally but sensitivity estimates for both RT-qPCR and VI increased by about 2\% (Table 4). In all models, the true median prevalence of AHSV infection was consistently estimated to be $>67 \%$ (clinically affected population) and $<0.3 \%$ (healthy population).

\subsection{Throughput and time estimates}

A maximum of 88 blood samples could be processed on a single extraction plate. The automated purification of nucleic acids of 88 blood samples took approximately $1 \mathrm{~h} 40 \mathrm{~min}$. The estimated "hands-on" time was limited to the setting up of the various regent plates and transferring of samples to the extraction plate and varied between $30 \mathrm{~min}$ and $1 \mathrm{~h}$. The automated nucleic acid extraction procedure took approximately $40 \mathrm{~min}$ and thus drastically increased the capacity and simultaneously reduced the necessary labour time. The AHSV RT-qPCR assay was completed in $<2 \mathrm{~h}$ and therefore a batch of samples could be processed within $4 \mathrm{~h}$ of arrival at the laboratory.

Table 4

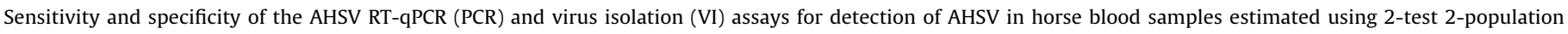
Bayesian latent class models.

\begin{tabular}{|c|c|c|c|}
\hline Model and priors & Test parameter & Median & 95\% probability interval \\
\hline \multicolumn{4}{|c|}{ 1. Dependence model with informative priors on VI specificity and prevalence in assumed AHSV-free population } \\
\hline \multirow[t]{4}{*}{ South African horses only } & PCR sensitivity & 0.978 & $0.708-0.9996$ \\
\hline & PCR specificity & 0.999 & $0.993-0.9999$ \\
\hline & VI sensitivity & 0.442 & $0.325-0.504$ \\
\hline & VI specificity & 0.9999 & $0.9996-1.0$ \\
\hline \multicolumn{4}{|c|}{ 2. Dependence model with informative priors on VI specificity and prevalence in assumed AHSV-free population } \\
\hline \multirow{3}{*}{ CVRL samples ( $n=98$ ) as healthy population } & PCR specificity & 0.993 & $0.963-0.9997$ \\
\hline & VI sensitivity & 0.437 & $0.322-0.504$ \\
\hline & VI specificity & 0.9996 & $0.9996-1.0$ \\
\hline \multicolumn{4}{|c|}{ 3. Independence model with non-informative beta $(1,1)$ priors } \\
\hline \multirow[t]{3}{*}{ South African horses only } & PCR sensitivity & 0.996 & $0.977-0.9998$ \\
\hline & PCR specificity & 0.999 & 0.993-0.9999 \\
\hline & VI sensitivity & 0.458 & $0.404-0.511$ \\
\hline
\end{tabular}




\section{Discussion}

The AHSV RT-qPCR assay described is unique amongst published assays in that it was designed using sequences from a wide variety of currently circulating field strains of AHSV (Quan et al., 2010). It also incorporates a proprietary synthetic external control to verify proper functioning of the assay components. The linear range, efficiency and limit of detection for this duplex assay were similar to that reported previously when the same primers and probes were incorporated into a duplex assay that also targeted the S8 gene of AHSV (Quan et al., 2010). Our results are also similar to those reported for other RT-qPCR's for AHSV (Agüero et al., 2008; Fernández-Pinero et al., 2008; Monaco et al., 2011). The intra- and inter-run repeatability for the AHSV component of the duplex assay was extremely good and were similar to those reported previously for a similar assay for the detection of Bluetongue virus (BTV) (Vandemeulebroucke et al., 2010). The XenoRNA EC component of the assay was also repeatable with CVs of less than $2 \%$ in positive and negative samples. The automated extraction system used in this study allowed the extraction and denaturing of dsRNA steps of the protocol to be completed concurrently, which resulted in a substantial increase in efficiency of sample processing as compared to the manual system used previously (Quan et al., 2010). This approach also reduced the number of steps in the protocol, thereby reducing opportunities for contamination. The incorporation of a robust automated nucleic acid extraction system into the assay substantially reduced processing time for each sample, making this assay suitable for high-throughput screening of samples from large groups of horses prior to movement or export or for surveillance during an AHS outbreak. The assay is complementary to new recombinant vaccines expressing outer capsid proteins of AHSV (VP2 and VP5) (Guthrie et al., 2009). Specifically, as the assay is specific for the AHSV S7 gene that encodes VP7, it can differentiate infected from vaccinated animals (DIVA), something that is not possible with the currently used live-attenuated (modified live) vaccines. The assay is also highly specific, detecting only AHSV and no other orbiviruses (Quan et al., 2010).

The diagnostic sensitivity and specificity of the AHSV RT-qPCR when it was applied to blood samples from AHS-suspect horses were close to perfect. In contrast, the sensitivity of virus isolation was estimated to be approximately $44 \%$. The results of this study confirm, therefore, that the sensitivity of the AHSV RTqPCR described is substantially greater than that of VI, which is considered currently to be the reference test for detection of AHSV. The severe nature of AHS and the implications of a falsenegative result necessitate the use of a diagnostic regulatory assay with high accuracy. Similar results have been reported for BTV, a virus closely related to AHSV, when RT-qPCR has been compared to VI (Vandemeulebroucke et al., 2010). It previously has been shown with BTV infection of ruminants that viral nucleic acid persists considerably longer in circulation than does infectious virus (MacLachlan et al., 1994, 2009), as also appears to be the case with AHSV in horses (Quan et al., 2010).

The latent class analysis approach was superior to specification of virus isolation as the reference test because the latter only allows calculation of relative sensitivity and specificity and the conclusion using that approach would have been that AHSV RT-qPCR was less specific than VI even though close to perfectly sensitive. Conclusions about the superiority of the sensitivity of AHSV RT-qPCR compared to VI were robust to choice of model type (dependence versus independence) and use of a smaller comparison population of AHSV-free horses from Dubai.

In summary, a high-throughput, analytically and diagnostically sensitive and specific RT-qPCR assay for the detection of AHSV is described. This assay is potentially highly useful and appropriate for demonstrating freedom or infection of horses with AHSV, thus it is appropriate that its reproducibility be evaluated in other laboratories as a global standard for detection of AHSV.

\section{Acknowledgements}

Racing South Africa (Pty) Ltd., The Mary Slack and Daughters Foundation and Thoroughbred Racing Trust of South Africa are thanked for their financial support for this study. Central Veterinary Research Laboratory, Dubai, United Arab Emirates for supply of blood samples from horses resident in an African horse sickness free country.

\section{References}

Anon., 2012. Manual of diagnostic tests and vaccines for terrestrial animals 2012: Chapter 1.1.5. Principles and methods of validation of diagnostic assays for infectious diseases. <http://www.oie.int/fileadmin/Home/eng/Health_standards/ tahm/1.01.05_VALIDATION.pdf > (accessed 14.09.12).

Anon., 2012. Manual of diagnostic tests and vaccines for terrestrial animals 2012: Chapter 2.5.1. African horse sickness. <http://www.oie .int/fileadmin/Home/eng/Health_standards/tahm/2.05.01_AHS.pdf> (accessed 14.09.12).

Anon., 2012. Terrestrial animal health code: Chapter 12.1. Infection with African horse sickness virus. <http://www.oie.int/index.php?id=169\&L=0\&htmfile= chapitre_1.12.1.htm> (accessed 14.09.12).

Agüero, M., Gómez-Tejedor, C., Cubillo, M.A., Rubio, C., Romero, E., Jiménez-Clavero, A., 2008. Real-time fluorogenic reverse transcription polymerase chain reaction assay for detection of African horse sickness virus. J. Vet. Diagn. Invest. 20, 325-328.

Bossuyt, P.M., Reitsma, J.B., Bruns, D.E., Gatsonis, C.A., Glasziou, P.P., Irwig, L.M., Lijmer, J.G., Moher, D., Rennie, D., de Vet, H.C.W., for the STARD Group, 2003. Towards complete and accurate reporting of studies of diagnostic accuracy: the STARD initiative. Clin. Chem. 49, 1-6.

Branscum, A.J., Gardner, I.A., Johnson, W.O., 2005. Estimation of diagnostic-test sensitivity and specificity through Bayesian modeling. Prev. Vet. Med. 68, 145-163.

Burns, M., Valdivia, H., 2008. Modelling the limit of detection in real-time quantitative PCR. Eur. Food Res. Technol. 226, 1513-1524.

Coetzer, J.A.W., Guthrie, A.J., 2004. African horse sickness. In: Coetzer, J.A.W., Tustin, R.C. (Eds.), Infectious Diseases of Livestock. Oxford University Press Southern Africa, Cape Town, pp. 1231-1246.

Fang, X., Willis, R.C., Burrell, A., Evans, K., Hoang, Q., Xu, W., Bounpheng, M., 2007. Automation of nucleic acid isolation on KingFisher magnetic particle processors. J. Assoc. Lab. Automat. 12, 195-201.

Fernández-Pinero, J., Fernández-Pacheco, P., Rodríguez, B., Sotelo, E., Robles, A., Arias, M., Sánchez-Vizcaíno, J.M., 2008. Rapid and sensitive detection of African horse sickness virus by real-time PCR. Res. Vet. Sci. 86, 353-358.

Gardner, I.A., Nielsen, S.S., Whittington, R.J., Collins, M.T., Bakker, D., Harris, B., Sreevatsan, S., Lombard, J.E., Sweeney, R., Smith, D.R., Gavalchin, J., Eda, S., 2011. Consensus-based reporting standards for diagnostic test accuracy studies for paratuberculosis in ruminants. Prev. Vet. Med. 101, 18-34.

Georgiadis, M.P., Johnson, W.O., Gardner, I.A., Singh, R., 2003. Correlation-adjusted estimation of sensitivity and specificity of two diagnostic tests. J. R. Stat. Soc. Ser. C: Appl. Stat. 52, 63-76.

Grubman, M.J., Lewis, S.A., 1992. Identification and characterization of the structural and nonstructural proteins of African horsesickness virus and determination of the genome coding assignments. Virology 186, 444-451.

Guthrie, A.J., Quan, M., 2009. African horse sickness. In: Mair, T.S., Hutchinson, R.E. (Eds.), Infectious Diseases of the Horse. Equine Veterinary Journal Ltd., Fordham, pp. 72-82.

Guthrie, A.J., Quan, M., Lourens, C.W., Audonnet, J.C., Minke, J.M., Yao, J., He, L., Nordgren, R., Gardner, I.A., MacLachlan, N.J., 2009. Protective immunization of horses with a recombinant canarypox virus vectored vaccine co-expressing genes encoding the outer capsid proteins of African horse sickness virus. Vaccine 27, 4434-4438.

Hamblin, C., Graham, S.D., Anderson, E.C., Crowther, J.R., 1990. A competitive ELISA for the detection of group-specific antibodies to African horse sickness virus. Epidemiol. Infect. 104, 303-312.

Hamblin, C., Mertens, P.P.C., Mellor, P.S., Burroughs, J.N., Crowther, J.R., 1991. A serogroup specific ELISA for the detection and identification of AHS virus. J. Virol. Methods 31, 285-292.

Howell, P.G., 1962. The isolation and identification of further antigenic types of African horsesickness virus. Onderstepoort J. Vet. Res. 29, 139-149.

Kweon, C.H., Kwon, B.J., Ko, Y.J., Kenichi, S., 2003. Development of competitive ELISA for serodiagnosis on African horsesickness virus using baculovirus expressed VP7 and monoclonal antibody. J. Virol. Methods 113, 13-18.

Laviada, M.D., Babin, M., Dominguez, J., Sánchez-Vizcaíno, J.M., 1992. Adaptation and field evaluation of an ELISA sandwich for detection of African horse sickness viruses. In: Walton, T.E., Osburn, B.I. (Eds.), Bluetongue, African Horse Sickness and Related Orbiviruses. CRC Press, Boca Raton, pp. 640-645.

Laviada, M.D., Sanchez-Vizcaino, J.M., Roy, P., Sobrino, F., 1997. Detection of African horsesickness virus by the polymerase chain reaction: a note. Invest. Agr.: Prod. Sanid. Anim. 12, 97-102. 
Lunn, D.J., Thomas, A., Best, N., Spiegelhalter, D., 2000. WinBUGS - A Bayesian modelling framework: concepts, structure, and extensibility. Stat. Comput. 10 , 325-337.

MacLachlan, N.J., Drew, C.P., Darpel, K.E., Worwa, G., 2009. The pathology and pathogenesis of bluetongue. J. Comp. Pathol. 141, 1-16.

MacLachlan, N.J., Guthrie, A.J., 2010. Re-emergence of bluetongue, African horse sickness, and other Orbivirus diseases. Vet. Res., 41.

MacLachlan, N.J., Nunamaker, R.A., Katz, J.B., Sawyer, M.M., Akita, G.Y., Osburn, B.I., Tabachnick, W.J., 1994. Detection of bluetongue virus in the blood of inoculated calves: comparison of virus isolation, PCR assay, and in vitro feeding of Culicoides variipennis. Arch. Virol. 136, 1-8.

Maree, S., Paweska, J.T., 2005. Preparation of recombinant African horse sickness virus VP7 antigen via a simple method and validation of a VP7-based indirect ELISA for the detection of group-specific IgG antibodies in horse sera. J. Virol. Methods 125, 55-65.

McIntosh, B.M., 1958. Immunological types of horsesickness virus and their significance in immunization. Onderstepoort J. Vet. Res. 27, 465-539.

Monaco, F., Polci, A., Lelli, R., Pinoni, C., Di Mattia, T., Mbulu, R.S., Scacchia, M., Savini, G., 2011. A new duplex real-time RT-PCR assay for sensitive and specific detection of African horse sickness virus. Mol. Cell. Probes 25, 87-93.
Quan, M., Lourens, C.W., MacLachlan, N.J., Gardner, I.A., Guthrie, A.J., 2010. Development and optimisation of a duplex real-time reverse transcription quantitative PCR assay targeting the VP7 and NS2 genes of African horse sickness virus. J. Virol. Methods 167, 45-52.

Quan, M., Van Vuuren, M., Howell, P.G., Groenewald, D., Guthrie, A.J., 2008. Molecular epidemiology of the African horse sickness virus S10 gene. J. Gen. Virol. 89, 1159-1168.

Roy, P., Mertens, P.P., Casal, I., 1994. African horse sickness virus structure. Comp. Immunol. Microbiol. Infect. Dis. 17, 243-273.

Vandemeulebroucke, E., De Clercq, K., Van der Stede, Y., Vandenbussche, F., 2010. A proposed validation method for automated nucleic acid extraction and RT-qPCR analysis: an example using Bluetongue virus. J. Virol. Methods 165, 76-82.

Wade-Evans, A.M., Woolhouse, T., O'Hara, R., Hamblin, C., 1993. The use of African horse sickness virus VP7 antigen, synthesised in bacteria, and anti-VP7 monoclonal antibodies in competitive ELISA. J. Virol. Methods 45, 179-188.

Zientara, S., Sailleau, C., Moulay, S., Plateau, E., Cruciere, C., 1993. Diagnosis and molecular epidemiology of the African horsesickness virus by the polymerase chain reaction and restriction patterns. Ann. Rech. Vet. 24, 385-395.

Zientara, S., Sailleau, C., Moulay, S., Wade-Evans, A., Cruciere, C., 1995. Application of the polymerase chain reaction to the detection of African horse sickness viruses. J. Virol. Methods 53, 47-54. 